Moroccan J. of Pure and Appl. Anal. (MJPAA)

Volume 4(2), 2018, Pages 77-84

ISSN: Online 2351-8227 - Print 2605-6364

DOI 10.1515/mjpaa-2018-0008

\title{
Weak solutions for generalized p-Laplacian systems via Young measures
}

Elhoussine Azroul ${ }^{1, a}$ ANd FARAh BALAAdich ${ }^{1, b}$

AвSTRACT. We prove the existence of weak solutions to a generalized p-Laplacian systems in degenerate form. The techniques of Young measure for elliptic systems are used to prove the existence result.

2010 Mathematics Subject Classification. Primary: 35J50, 35J57, 35D30, 28Axx.

Key words and phrases. $p$-Laplacian systems, Weak solutions, Young measures.

\section{Introduction}

We study the existence of weak solutions $u: \Omega \rightarrow \mathbb{R}^{m}, m \in \mathbb{N}$, to the following Dirichlet problem:

$$
\left\{\begin{array}{rll}
-\operatorname{div}(\phi(D u-\Theta(u))) & =f & \text { in } \Omega, \\
u & =0 & \text { on } \partial \Omega,
\end{array}\right.
$$

with

$$
\phi(A)=|A|^{p-2} A, \quad \forall A \in \mathbb{M}^{m \times n} .
$$

Here $\Omega$ is a bounded open domain in $\mathbb{R}^{n}(n \geq 2)$ and $\mathbb{M}^{m \times n}$ is the set of $m \times n$ real matrices with reduced $\mathbb{R}^{m n}$ topology, that is, if $A \in \mathbb{M}^{m \times n}$, then $|A|$ is the norm of $A$ when regarded as a vector in $\mathbb{R}^{m n} . \mathbb{M}^{m \times n}$ is endowed with the scalar product $A: B=A_{i j} B_{i j}$ (with the usual summation convention). We assume that $p$ is a real number such that $p \in(1, \infty)$. The source term $f$ is supposed lying in $W^{-1, p^{\prime}}\left(\Omega ; \mathbb{R}^{m}\right)$ the dual space of $W_{0}^{1, p}\left(\Omega ; \mathbb{R}^{m}\right)$ and $\Theta: \mathbb{R}^{m} \rightarrow \mathbb{M}^{m \times n}$ is a continuous function satisfies

$$
\Theta(0)=0 \text { and }|\Theta(\xi)-\Theta(\eta)| \leq c|\xi-\eta|
$$

Received February 14, 2019 - Accepted April 3, 2019.

(C) The Author(s) 2017. This article is published with open access by Sidi Mohamed Ben Abdallah University.

${ }^{1}$ Department of Mathematics, Faculty of Sciences Dhar El Mehraz, B.P. 1796 Atlas, Fez-Morocco.

a e-mail: elhoussine.azroul@gmail.com

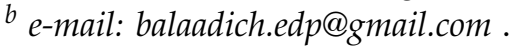


for all $\xi, \eta \in \mathbb{R}^{m}$, where $c$ is a positive constant related to the exponent $p$ and the diameter of $\Omega(\operatorname{diam}(\Omega))$ by the following:

$$
c<\frac{1}{\operatorname{diam}(\Omega)}\left(\frac{1}{2}\right)^{\frac{1}{p}}
$$

For several decades, there have been intensive research activities for equations, or systems, of $p$-Laplacian type. In [12], several examples of degenerate elliptic equations are presented. The author proved the existence of a weak solution by various methods. Dibenedetto and Manfredi [4] considered the following nonlinear elliptic system $\left.\operatorname{div}\left(|D u|^{p-2} D u\right)=\operatorname{div}\left(|F|^{p-2} D\right)\right)$, for $F \in L_{l o c}^{p}\left(\Omega ; \mathbb{R}^{m}\right)$ and proved the existence of local weak solution and some estimates of $D u$ in $\left[B M O_{l o c}(\Omega)\right]^{N m}$. In [10], the authors proved a regularity result for the quasilinear equation $\operatorname{div}\left((A D u \cdot D u)^{\frac{(p-2)}{2}} A D u\right)=\operatorname{div}\left(|F|^{p-2} F\right)$. They studied the regularity of $F$ which reflected to the solutions under minimal assumptions on the coefficient matrix $A$. A collect of some very recent pointwise bounds for the gradient of solutions, and the solutions themselves, to the $p$-Laplace system with right hand side in divergence form were discussed in [3].

In view of [9], our system $-\operatorname{div}\left(|D u-\Theta(u)|^{p-2}(D u-\Theta(u))\right)=f$ is a nonlinear degenerate and singular elliptic system according to the cases $p>2$ and $1<p<2$, respectively.

In the present paper, due to the term $\Theta$ in Eq. (1.1), we don't have such Leray-Lions conditions and we can't use the main techniques as in [10]. Our aim here is to prove the existence of weak solutions by using the concept of Young measures as technical tool to describe the weak limits of a sequence of approximating solutions. This concept can be used when weak convergence does not behave as one desire with respect to nonlinear operators.

We say that $u \in W_{0}^{1, p}\left(\Omega ; \mathbb{R}^{m}\right)$ is a weak solution to (1.1) if

$$
\begin{aligned}
\int_{\Omega} \phi(D u-\Theta(u)): D \varphi d x & :=\int_{\Omega}|D u-\Theta(u)|^{p-2}(D u-\Theta(u)): D \varphi d x \\
& =\langle f, \varphi\rangle
\end{aligned}
$$

holds for all $\varphi \in W_{0}^{1, p}\left(\Omega ; \mathbb{R}^{m}\right)$. Here $\langle.,$.$\rangle denote the duality pairing between W^{-1, p^{\prime}}\left(\Omega ; \mathbb{R}^{m}\right)$ and $W_{0}^{1, p}\left(\Omega ; \mathbb{R}^{m}\right)$.

The main result of this paper is the following:

Theorem 1.1. Suppose that $\Theta$ satisfies (1.2), then there exists at least one weak solution of the problem (1.1).

\section{Preliminaries}

Let $\Omega$ be a bounded open in $\mathbb{R}^{n}, n \geq 2$, with smooth boundary $\partial \Omega$. Let $1<p<\infty$. Throughout this paper, we will use the following Poincaré's inequality (see [11, Lemma 2.2]), there exists a positive constant $\alpha=\operatorname{diam}(\Omega)$ such that

$$
\|v\|_{p} \leq \frac{\alpha}{2}\|D v\|_{p}, \quad v \in W_{0}^{1, p}\left(\Omega ; \mathbb{R}^{m}\right)
$$

The relation (2.1) and the Hölder inequality are central to establish the required estimates to prove the desired results.

We recall the following useful lemma:

Lemma 2.1 ([1]). Let $\xi, \eta \in \mathbb{R}^{m}$ and let $1<p<\infty$. We have

$$
\frac{1}{p}|\xi|^{p}-\frac{1}{p}|\eta|^{p} \leq|\xi|^{p-2} \xi \cdot(\xi-\eta)
$$

As mentioned in the introduction, we will use the concept of Young measure. Here, we give a brief review on Young measure and some properties needed in this paper.

By $\mathcal{C}_{0}\left(\mathbb{R}^{m}\right)$ we denote the closure of the space of continuous functions on $\mathbb{R}^{m}$ with compact support with respect to the $\|\cdot\|_{\infty}$-norm. Its dual space can be identified with $\mathcal{M}\left(\mathbb{R}^{m}\right)$, the space of signed Radon measures with finite mass. The related duality pairing is given by

$$
\langle v, \varphi\rangle=\int_{\mathbb{R}^{m}} \varphi(\lambda) d v(\lambda) .
$$


Note that $\langle v, i d\rangle=\int_{\mathbb{R}^{m}} \lambda d v(\lambda)$.

Lemma 2.2. [7, Theorem 1.5.2] Let $\left\{z_{j}\right\}_{j \geq 1}$ be a bounded sequence in $L^{\infty}\left(\Omega ; \mathbb{R}^{m}\right)$. Then there exists a subsequence $\left\{z_{k}\right\}_{k} \subset\left\{z_{j}\right\}_{j}$ and a Borel probability measure $v_{x}$ on $\mathbb{R}^{m}$ for a.e. $x \in \Omega$, such that for almost each $\varphi \in \mathcal{C}\left(\mathbb{R}^{m}\right)$ we have

$$
\varphi\left(z_{k}\right) \rightarrow^{*} \bar{\varphi} \text { weakly in } L^{\infty}\left(\Omega ; \mathbb{R}^{m}\right),
$$

where $\bar{\varphi}(x)=\left\langle v_{x}, \varphi\right\rangle=\int_{\mathbb{R}^{m}} \varphi(\lambda) d v_{x}(\lambda)$ for a.e. $x \in \Omega$.

We call $\left\{v_{x}\right\}_{x \in \Omega}$ the family of Young measures associated with the subsequence $\left\{z_{k}\right\}_{k \geq 1}$.

The fundamental theorem on Young measure can be stated in the following lemma:

Lemma 2.3. [5] Let $\Omega \subset \mathbb{R}^{n}$ be Lebesgue measurable (not necessarily bounded) and $z_{j}: \Omega \rightarrow \mathbb{R}^{m}, j=1, .$. , be a sequence of Lebesgue measurable functions. Then there exists a subsequence $z_{k}$ and a family $\left\{v_{x}\right\}_{x \in \Omega}$ of non-negative Radon measures on $\mathbb{R}^{m}$, such that

(i) $\left\|v_{x}\right\|_{\mathcal{M}\left(\mathbb{R}^{m}\right)}:=\int_{\mathbb{R}^{m}} d v_{x} \leq 1$ for almost $x \in \Omega$.

(ii) $\varphi\left(z_{k}\right) \rightarrow^{*} \bar{\varphi}$ weakly* in $L^{\infty}(\Omega)$ for all $\varphi \in \mathcal{C}_{0}\left(\mathbb{R}^{m}\right)$, where $\bar{\varphi}(x)=\left\langle v_{x}, \varphi\right\rangle$ and $\mathcal{C}_{0}\left(\mathbb{R}^{m}\right)=\left\{\varphi \in \mathcal{C}\left(\mathbb{R}^{m}\right)\right.$ : $\left.\lim _{|z| \rightarrow \infty}|\varphi(z)|=0\right\}$.

(iii) If for all $R>0$

$$
\lim _{L \rightarrow \infty} \sup _{k}\left|\left\{x \in \Omega \cap B_{R}(0):\left|z_{k}(x)\right| \geq L\right\}\right|=0,
$$

then $\left\|v_{x}\right\|=1$ for almost every $x \in \Omega$, and for all measurable $\Omega^{\prime} \subset \Omega$ there holds $\varphi\left(z_{k}\right) \rightarrow \bar{\varphi}=\left\langle v_{x}, \varphi\right\rangle$ weakly in $L^{1}\left(\Omega^{\prime}\right)$ for continuous function $\varphi$ provided the sequence $\varphi\left(z_{k}\right)$ is weakly precompact in $L^{1}\left(\Omega^{\prime}\right)$.

\section{Galerkin approximation and priori estimates}

Let define $T: W_{0}^{1, p}\left(\Omega ; \mathbb{R}^{m}\right) \rightarrow W^{-1, p^{\prime}}\left(\Omega ; \mathbb{R}^{m}\right)$ in the following way

$$
\langle T(u), \varphi\rangle=\int_{\Omega} \phi(D u-\Theta(u)): D \varphi d x-\langle f, \varphi\rangle .
$$

Our problem (1.1)-(1.2) is then equivalent to find $u \in W_{0}^{1, p}\left(\Omega ; \mathbb{R}^{m}\right)$ such that $\langle T(u), \varphi\rangle=0$ for all $\varphi \in W_{0}^{1, p}\left(\Omega ; \mathbb{R}^{m}\right)$.

Lemma 3.1. We have the following properties:

(i) $T: W_{0}^{1, p}\left(\Omega ; \mathbb{R}^{m}\right) \rightarrow W^{-1, p^{\prime}}\left(\Omega ; \mathbb{R}^{m}\right)$ is linear, well defined and bounded.

(ii) The restriction of $T$ to a finite linear subspace of $W_{0}^{1, p}\left(\Omega ; \mathbb{R}^{m}\right)$ is continuous.

(iii) $T$ is coercive.

Proof. (i) $T$ is trivially linear. For arbitrary $u \in W_{0}^{1, p}\left(\Omega ; \mathbb{R}^{m}\right)$, by the Hölder's inequality, Poincarés inequality and the Eq. (1.2), we have

$$
\begin{aligned}
|\langle T(u), \varphi\rangle| & =\left|\int_{\Omega} \phi(D u-\Theta(u)): D \varphi d x-\langle f, \varphi\rangle\right| \\
& \leq \int_{\Omega}|D u-\Theta(u)|^{p-1}|D \varphi| d x+\|f\|_{-1, p^{\prime}}\|\varphi\|_{1, p} \\
& \leq\left(\int_{\Omega}|D u-\Theta(u)|^{p} d x\right)^{\frac{1}{p^{\prime}}}\|D \varphi\|_{p}+\|f\|_{-1, p^{\prime}}\|\varphi\|_{1, p} \\
& \leq 2^{\frac{(p-1)^{2}}{p}}\left(\|D u\|_{p}^{p}+\|\Theta(u)\|_{p}^{p}\right)^{\frac{p-1}{p}}\|D \varphi\|_{p}+\|f\|_{-1, p^{\prime}}\|\varphi\|_{1, p} \\
& \leq c^{\prime}\|\varphi\|_{1, p}
\end{aligned}
$$

for some positive constant $c^{\prime}$. In the above inequality we have used

$$
|a+b|^{p} \leq 2^{p-1}\left(|a|^{p}+|b|^{p}\right) \quad(p>1) .
$$


It follows that $T$ is well defined and bounded.

(ii) Let $V$ be a subspace of $W_{0}^{1, p}\left(\Omega ; \mathbb{R}^{m}\right)$ with $\operatorname{dim} V=r$ and $\left(e_{i}\right)_{i=1}^{r}$ a basis of $V$. Let $\left(u_{k}=a_{k}^{i} e_{i}\right)$ be a sequence in $V$ which converges to $u=a^{i} e_{i}$ in $V$ (with conventional summation). Then $u_{k} \rightarrow u$ and $D u_{k} \rightarrow D u$ almost everywhere for a subsequence still denoted by $\left\{u_{k}\right\}$. On the other hand, $\left\|u_{k}\right\|_{p}$ and $\left\|D u_{k}\right\|_{p}$ are bounded by a constant $C$. Indeed, since $u_{k} \rightarrow u$ strongly in $V$,

$$
\int_{\Omega}\left|u_{k}-u\right|^{p} d x \rightarrow 0 \text { and } \int_{\Omega}\left|D u_{k}-D u\right|^{p} d x \rightarrow 0
$$

then there exists a subsequence of $\left\{u_{k}\right\}$ still denoted by $\left\{u_{k}\right\}$ and $g_{1}, g_{2} \in L^{1}(\Omega)$ such that $\left|u_{k}-u\right|^{p} \leq g_{1}$ and $\left|D u_{k}-D u\right|^{p} \leq g_{2}$. According to (3.1), it follows that

$$
\begin{aligned}
\left|u_{k}\right|^{p}=\left|u_{k}-u+u\right|^{p} & \leq 2^{p-1}\left(\left|u_{k}-u\right|^{p}+|u|^{p}\right) \\
& \leq 2^{p-1}\left(g_{1}+|u|\right) .
\end{aligned}
$$

Similarly

$$
\left|D u_{k}\right|^{p} \leq 2^{p-1}\left(g_{2}+|D u|^{p}\right)
$$

By the continuity of the function $\Theta$, it follows that

$$
\phi\left(D u_{k}-\Theta\left(u_{k}\right)\right): D \varphi \rightarrow \phi(D u-\Theta(u)): D \varphi \quad \text { almost everywhere. }
$$

Let $\Omega^{\prime} \subset \Omega$ be a measurable subset and $\varphi \in W_{0}^{1, p}\left(\Omega ; \mathbb{R}^{m}\right)$. As in the proof of the property $(i)$, we obtain

$$
\begin{aligned}
& \int_{\Omega^{\prime}} \mid \phi\left(D u_{k}-\Theta\left(u_{k}\right)\right): D \varphi \mid d x \\
& \leq 2^{\frac{(p-1)^{2}}{p}}(\underbrace{\left\|D u_{k}\right\|_{p}^{p}}_{\leq C}+c^{p} \underbrace{\left\|u_{k}\right\|_{p}^{p}}_{\leq C})^{\frac{p-1}{p}}\left(\int_{\Omega^{\prime}}|D \varphi|^{p} d x\right)^{\frac{1}{p}} .
\end{aligned}
$$

Since $\int_{\Omega^{\prime}}|D \varphi|^{p} d x$ is arbitrary small if the measure of $\Omega^{\prime}$ is chosen small enough, then $\left(\phi\left(D u_{k}-\Theta\left(u_{k}\right)\right): D \varphi\right)$ is equiintegrable. Applying the Vitali Theorem, it follows that $T$ is continuous.

(iii) We have

$$
\langle T(u), u\rangle=\int_{\Omega}|D u-\Theta(u)|^{p-2}(D u-\Theta(u)): D u d x-\langle f, u\rangle .
$$

By Lemma 2.1, we have

$$
|F|^{p-2} F:(F-G) \geq \frac{1}{p}|F|^{p}-\frac{1}{p}|G|^{p},
$$

then by taking $F=D u-\Theta(u)$ and $G=-\Theta(u),(F-G=D u)$, we obtain

$$
\begin{aligned}
\mid D u-\Theta(u) & \left.\right|^{p-2}(D u-\Theta(u)): D u \\
& =|D u-\Theta(u)|^{p-2}(D u-\Theta(u)):(D u-\Theta(u)+\Theta(u)) \\
& \geq \frac{1}{p}|D u-\Theta(u)|^{p}-\frac{1}{p}|\Theta(u)|^{p} .
\end{aligned}
$$

By vertue of (3.2), we deduce that

$$
\langle T(u), u\rangle \geq \frac{1}{p} \int_{\Omega}|D u-\Theta(u)|^{p} d x-\frac{1}{p} \int_{\Omega}|\Theta(u)|^{p} d x-\|f\|_{-1, p^{\prime}}\|u\|_{1, p} .
$$

We have

$$
\begin{aligned}
\frac{1}{2^{p-1}}|D u|^{p} & =\frac{1}{2^{p-1}}|D u-\Theta(u)+\Theta(u)|^{p} \\
& \leq \frac{1}{2^{p-1}}\left[2^{p-1}\left(|D u-\Theta(u)|^{p}+|\Theta(u)|^{p}\right)\right] \\
& =|D u-\Theta(u)|^{p}+|\Theta(u)|^{p}
\end{aligned}
$$


implies that

$$
\begin{aligned}
\langle T(u), u\rangle & \geq \frac{1}{p} \int_{\Omega}\left(\frac{1}{2^{p-1}}|D u|^{p}-|\Theta(u)|^{p}\right) d x-\frac{1}{p} \int_{\Omega}|\Theta(u)|^{p} d x-\|f\|_{-1, p^{\prime}}\|u\|_{1, p} \\
& \geq \frac{1}{p 2^{p-1}} \int_{\Omega}|D u|^{p} d x-\frac{2}{p} \int_{\Omega}|\Theta(u)|^{p} d x-\|f\|_{-1, p^{\prime}}\|u\|_{1, p} \\
& \geq \frac{1}{p 2^{p-1}} \int_{\Omega}|D u|^{p} d x-\frac{1}{p 2^{p}} \int_{\Omega}|D u|^{p} d x-\|f\|_{-1, p^{\prime}}\|u\|_{1, p} \\
& =\frac{1}{p 2^{p}} \int_{\Omega}|D u|^{p} d x-\|f\|_{-1, p^{\prime}}\|u\|_{1, p} .
\end{aligned}
$$

Consequently, $T$ is coercive.

To prove Theorem 1.1, we will apply a Galerkin schema. Let $V_{1} \subset V_{2} \subset \ldots \subset W_{0}^{1, p}\left(\Omega ; \mathbb{R}^{m}\right)$ be a sequence of finite dimensional subspaces with the property that $\cup_{k \geq 1} V_{k}$ is dense in $W_{0}^{1, p}\left(\Omega ; \mathbb{R}^{m}\right)$. Note that the existence of $\left(V_{k}\right)$ is guaranteed by the separability of $W_{0}^{1, p}\left(\Omega ; \mathbb{R}^{m}\right)$.

Now, we can construct the approximating solutions:

Lemma 3.2. (i) For all $k \in \mathbb{N}$ there exists $u_{k} \in V_{k}$ such that

$$
\left\langle T\left(u_{k}\right), \varphi\right\rangle=0 \text { for all } \varphi \in V_{k} .
$$

(ii) There exists a constant $R>0$ such that

$$
\left\|u_{k}\right\|_{1, p} \leq R \quad \text { for all } k \in \mathbb{N} .
$$

Proof. (i) Let fix $k$ and assume that $\operatorname{dim} V_{k}=r$. For simplicity, we write $\sum_{1 \leq i \leq r} a^{i} e_{i}=a^{i} e_{i}$ where $\left(e_{i}\right)_{i=1}^{r}$ is a basis of $V_{k}$. Define the map

$$
\begin{aligned}
S: \mathbb{R}^{r} & \longrightarrow \mathbb{R}^{r} \\
\left(a^{1}, . ., a^{r}\right) & \longrightarrow\left(\left\langle T\left(a^{i} e_{i}\right), e_{j}\right\rangle\right)_{j=1, \ldots, r} .
\end{aligned}
$$

Remark that $S$ is continuous by Lemma 3.1(ii). Let $a \in \mathbb{R}^{r}$ and $u=a^{i} e_{i} \in V_{k}$, then $\|a\|_{\mathbb{R}^{r}} \rightarrow \infty$ is equivalent to $\|u\|_{1, p} \rightarrow \infty$ and

$$
S(a) \cdot a=\langle T(u), u\rangle
$$

Hence, by Lemma 3.1(iii), we have

$$
S(a) \cdot a \rightarrow \infty \quad \text { as } \quad\|a\|_{\mathbb{R}^{r}} \rightarrow \infty .
$$

Thus, there exists $R>0$ such that for all $a \in \partial B_{R}(0) \subset \mathbb{R}^{r}$ we have $S(a) \cdot a>0$. According to the usual topological arguments [13, Proposition 2.8], $S(x)=0$ has a solution $x \in B_{R}(0)$. Hence, for all $k$ there exists $u_{k} \in V_{k}$ such that

$$
\left\langle T\left(u_{k}\right), \varphi\right\rangle=0 \text { for all } \varphi \in V_{k} .
$$

(ii) Since $\langle T(u), u\rangle \rightarrow \infty$ as $\|u\|_{1, p} \rightarrow \infty$, it follows that there exists $R>0$ with the property, that $\langle T(u), u\rangle>$ 1 whenever $\|u\|_{1, p}>R$. Consequently, for the sequence of Galerkin approximations $u_{k} \in V_{k}$ which satisfy $\left\langle T\left(u_{k}\right), u_{k}\right\rangle=0$ by (3.3), we have the uniform bound

$$
\left\|u_{k}\right\|_{1, p} \leq R \quad \text { for all } k \in \mathbb{N} .
$$




\section{Passage to the limit}

This section is devoted first to identify weak limits of gradient sequences by means of the Young measures and then we pass to the limit in the approximating equations. The sequence (or at least a subsequence) of the gradients $D u_{k}$ generates a Young measure $v_{x}$. Now, we collect some facts about the Young measure $v=\left\{v_{x}\right\}_{x}$ in the following lemma:

Lemma 4.1. Let $\left(u_{k}\right)$ the sequence defined in Lemma 3.2. Then the Young measure $v_{x}$ generated by $D u_{k}$ in $L^{p}\left(\Omega ; \mathbb{M}^{m \times n}\right)$ has the following properties:

(i) $v_{x}$ is a probability measure, i.e. $\left\|v_{x}\right\|_{\mathcal{M}\left(\mathbb{M}^{m \times n}\right)}=1$ for almost every $x \in \Omega$.

(ii) The weak $L^{1}$-limit of $D u_{k}$ is given by $\left\langle v_{x}, i d\right\rangle=\int_{\mathbb{M}^{m \times n}} \lambda d v_{x}(\lambda)$.

(iii) $v_{x}$ satisfies $\left\langle v_{x}, i d\right\rangle=D u(x)$ for almost every $x \in \Omega$.

Proof. (i) Let $v_{x}$ the Young measure generated by $D u_{k}$ (see Lemma 2.2). Since $\left(u_{k}\right)$ is bounded in $W_{0}^{1, p}\left(\Omega ; \mathbb{R}^{m}\right)$ by (3.4), then there exists a constant $C \geq 0$ such that for any $R>0$,

$$
\begin{aligned}
C \geq \int_{\Omega}\left|D u_{k}\right|^{p} d x & \geq \int_{\left\{x \in \Omega \cap B_{R}(0):\left|D u_{k}\right| \geq L\right\}}\left|D u_{k}\right|^{p} d x \\
& \geq L^{p}\left|\left\{x \in \Omega \cap B_{R}(0):\left|D u_{k}\right| \geq L\right\}\right| .
\end{aligned}
$$

Hence

$$
\sup _{k \in \mathbb{N}}\left|\left\{x \in \Omega \cap B_{R}(0):\left|D u_{k}\right| \geq L\right\}\right| \leq \frac{C}{L^{p}} \rightarrow 0 \quad \text { as } L \rightarrow \infty .
$$

According to Lemma 2.2(iii), it follows that $\left\|v_{x}\right\|_{\mathcal{M}\left(\mathbb{M}^{m \times n}\right)}=1$ for almost every $x \in \Omega$.

(ii) Since $L^{p}\left(\Omega ; M^{m \times n}\right)$ is reflexive $\left(p>1\right.$ and $\left.\mathbb{M}^{m \times n} \cong \mathbb{R}^{m n}\right)$ and in view of (3.4), we deduce the existence of a subsequence (still denoted by $D u_{k}$ ) weakly convergent in $L^{p}\left(\Omega ; \mathbb{M}^{m \times n}\right)$. Moreover, weakly convergent in $L^{1}\left(\Omega ; \mathbb{M}^{m \times n}\right)$. By taking $\varphi$ as the identity mapping $I$ in Lemma 2.2(iii), we have

$$
D u_{k} \rightarrow\left\langle v_{x}, i d\right\rangle=\int_{\mathbb{M}^{m \times n}} \lambda d v_{x}(\lambda) \text { weakly in } L^{1}\left(\Omega ; \mathbb{M}^{m \times n}\right) .
$$

(iii) By the equation (3.4), a subsequence of $\left\{u_{k}\right\}$ converges weakly in $W_{0}^{1, p}\left(\Omega ; \mathbb{R}^{m}\right)$ to an element denoted by $u$. Thus $u_{k} \rightarrow u$ in $L^{p}\left(\Omega ; \mathbb{R}^{m}\right)$ and $D u_{k} \rightarrow D u$ in $L^{p}\left(\Omega ; \mathbb{M}^{m \times n}\right)$ (for a subsequence). Owing to (ii), the uniquenesses of the limit implies that

$$
\left\langle v_{x}, i d\right\rangle=D u(x) \text { for a.e. } x \in \Omega \text {. }
$$

Now, we have all ingredients to pass to the limit in the approximating equations and to prove Theorem 1.1. Let $\left(u_{k}\right)$ be the sequence constructed in Lemma 3.2.

Proof of Theorem 1.1. Let start by proving that $u_{k} \rightarrow u$ in measure. By (3.4), we have for a subsequence $u_{k} \rightarrow u$ in $L^{p}\left(\Omega ; \mathbb{R}^{m}\right)$. Let $E_{k, \epsilon}=\left\{x:\left|u_{k}(x)-u(x)\right| \geq \epsilon\right\}$, then

$$
\int_{\Omega}\left|u_{k}(x)-u(x)\right|^{p} d x \geq \int_{E_{k, \epsilon}}\left|u_{k}(x)-u(x)\right|^{p} d x \geq \epsilon^{p}\left|E_{k, \epsilon}\right|
$$

which implies

$$
\left|E_{k, \epsilon}\right| \leq \frac{1}{\epsilon^{p}} \int_{\Omega}\left|u_{k}(x)-u(x)\right|^{p} d x \rightarrow 0 \quad \text { as } k \rightarrow \infty .
$$

Therefore, $u_{k} \rightarrow u$ in measure for $k \rightarrow \infty$, and we may infer that, after extraction of a suitable subsequence, if necessary,

$$
u_{k} \rightarrow u \text { almost everywhere for } k \rightarrow \infty \text {. }
$$

According to a weak limit defined in Lemma 4.1 and the continuity of $\Theta$, we can write

$$
D u_{k}-\Theta\left(u_{k}\right) \rightarrow \int_{\mathbb{M}^{m \times n}}(\lambda-\Theta(u)) d v_{x}(\lambda)
$$




$$
\begin{aligned}
& =\int_{\mathbb{M}^{m \times n}} \lambda d v_{x}(\lambda)-\Theta(u) \underbrace{\int_{\mathbb{M}^{m \times n}} d v_{x}(\lambda)}_{:=1} \\
& =D u-\Theta(u)
\end{aligned}
$$

weakly in $L^{1}(\Omega)$, since $\left(D u_{k}-\Theta\left(u_{k}\right)\right)$ is equiintegrable by (1.2). Therefore

$$
\left|D u_{k}-\Theta\left(u_{k}\right)\right|^{p-2}\left(D u_{k}-\Theta\left(u_{k}\right)\right) \rightarrow|D u-\Theta(u)|^{p-2}(D u-\Theta(u))
$$

weakly in $L^{1}(\Omega)$. Since $L^{p}(\Omega)$ is reflexive and $\phi\left(D u_{k}-\Theta\left(u_{k}\right)\right)$ is bounded (by (3.1)), the sequence $\left\{\phi\left(D u_{k}-\right.\right.$ $\left.\left.\Theta\left(u_{k}\right)\right)\right\}$ converges in $L^{p^{\prime}}(\Omega)$. Hence its weak $L^{p^{\prime}}$-limit is also $\phi(D u-\Theta(u))$.

We may infer that

$$
\lim _{k \rightarrow \infty} \int_{\Omega} \phi\left(D u_{k}-\Theta\left(u_{k}\right)\right): D \varphi(x) d x=\int_{\Omega} \phi(D u-\Theta(u)): D \varphi(x) d x \forall \varphi \in \cup_{k \geq 1} V_{k} .
$$

For any $v \in W_{0}^{1, p}\left(\Omega ; \mathbb{R}^{m}\right)$, since $\underset{k \geq 1}{\cup} V_{k}$ is dense in $W_{0}^{1, p}\left(\Omega ; \mathbb{R}^{m}\right)$, there is a sequence $\left\{v_{k}\right\} \subset \underset{k \geq 1}{\cup} V_{k}$ such that $v_{k} \rightarrow v$ in $W_{0}^{1, p}\left(\Omega ; \mathbb{R}^{m}\right)$ as $k \rightarrow \infty$. Since

$$
\int_{\Omega}\left(\phi\left(D u_{k}-\Theta\left(u_{k}\right)\right): D v-\phi(D u-\Theta(u)): D v\right) d x \rightarrow 0 \text { as } k \rightarrow \infty,
$$

we have

$$
\begin{aligned}
& \left\langle T\left(u_{k}\right), v_{k}\right\rangle-\langle T(u), v\rangle \\
& =\int_{\Omega}\left(\phi\left(D u_{k}-\Theta\left(u_{k}\right)\right): D v_{k}-\phi(D u-\Theta(u)): D v\right) d x-\left\langle f, v_{k}-v\right\rangle \\
& =\int_{\Omega}\left(\phi\left(D u_{k}-\Theta\left(u_{k}\right)\right):\left(D v_{k}-D v\right)+\left(\phi\left(D u_{k}-\Theta\left(u_{k}\right)\right)-\phi(D u-\Theta(u))\right): D v\right) d x \\
& -\left\langle f, v_{k}-v\right\rangle .
\end{aligned}
$$

The right hand side of the above equation tends to 0 as $k \rightarrow \infty$. By vertue of Lemma 3.2(i), it follows that $\langle T(u), v\rangle=0$ for all $v \in W_{0}^{1, p}\left(\Omega ; \mathbb{R}^{m}\right)$.

\section{References}

1. A. Abassi, A. El Hachimi, A. Jamea, Entropy solutions to nonlinear Neumann problems with $L^{1}$-data, Int. J. Math. Statist. 2 (2008) 4-17.

2. J. M. Ball, A version of the fundamental theorem for Young measures. In: PDEs and Continuum Models of Phase Transitions (Nice, 1988). Lecture Notes in Phys, 344 (1989) 207-215.

3. D. Breit, A. Cianchi, L. Diening, T. Kuusi, S. Schwarzaker, The p-Laplace system with right-hand side in divergence form: inner and up to the boundary pointwise estimates, Nonlinear Analysis: Theory, Methods \& Applications, 115(2017) 200-212.

4. E. DiBenedetto, J. Manfredi, On the Higher Integrability of the Gradient of Weak Solutions of Certain Degenerate Elliptic Systems, American Journal of Mathematics, 115 (5) (1993) 1107-1134.

5. G. Dolzmann, N. Hungerbühler, S. Müller, Nonlinear elliptic systems with measure valued right hand side. Math. Z, 226 (1997) 545-574.

6. F. Duzaar, G. Mingione, Local Lipschitz regularity for degenerate elliptic systems, Ann. I. H. Poincaré AN 27 (2010) 1361-1396.

7. LC. Evans, Weak Convergence Methods for Nonlinear Partial Differential Equations. Am. Math. Soc., New York 1990.

8. N. Hungerbühler, A refinement of Ball's theorem on Young measures, N.Y. J. Math, 3 (1997) 48-53.

9. T. Iwaniec, Projections onto gradients fields and $L^{p}$-estimates for degenrated elliptic operators, Studia Mathematica, 75 (3) (1983) 293-312. 
10. J. Kinuunen, S. Zhou, A local estimates for nonlinear equations with discontinuous coefficients, Commun. Partial Differential Equations 24 (11-12) (1999) 2043-2068.

11. G M. Lieberman, The natural generalizationj of the natural conditions of ladyzhenskaya and uraltseva for elliptic equations, Commun. In. Partial Differential Equations. 16(2\&3) (1991) 311-361.

12. M. Struwe, Variational Methods, Springer Verlag Berlin, Heidelberg, New York, second edition 1996.

13. E. Zeidler, Nonlinear functional analysis and its application, volume I. Springer, 1986. 